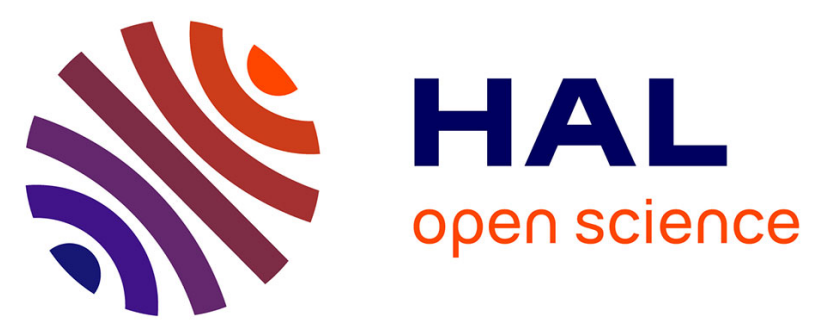

\title{
DES DISPOSITIFS POUR LES ÉLÈVES PERTURBATEURS : LES COLLÈGES À L'HEURE DE LA SOUS-TRAITANCE?
}

Benjamin Moignard, Stéphanie Rubi

\section{- To cite this version:}

Benjamin Moignard, Stéphanie Rubi. DES DISPOSITIFS POUR LES ÉLÈVES PERTURBATEURS : LES COLLĖGES À L'HEURE DE LA SOUS-TRAITANCE?. Carrefours de l'éducation, 2013, Les établissements scolaires à l'heure des "dispositifs", 36 (2), 10.3917/cdle.036.0047 . hal-01224700

\section{HAL Id: hal-01224700 \\ https://hal.science/hal-01224700}

Submitted on 6 Nov 2015

HAL is a multi-disciplinary open access archive for the deposit and dissemination of scientific research documents, whether they are published or not. The documents may come from teaching and research institutions in France or abroad, or from public or private research centers.
L'archive ouverte pluridisciplinaire HAL, est destinée au dépôt et à la diffusion de documents scientifiques de niveau recherche, publiés ou non, émanant des établissements d'enseignement et de recherche français ou étrangers, des laboratoires publics ou privés. 
Des dispositifs pour les élèves perturbateurs : les collèges à l'heure de la sous-traitance ?

Benjamin Moignard ${ }^{1,2}$

benjamin.moignard@u-pec.fr

Stéphanie Rubi ${ }^{2,3}$

stefrubi@gmail.com

${ }^{1}$ Centre interdisciplinaire de recherche "Culture, Éducation, Formation, Travail" (CIRCEFT EA 4384) - Université Paris-Est

${ }^{2}$ Observatoire Universitaire International Education et Prévention (OUIEP)

${ }^{3}$ Laboratoire Cultures Éducation Sociétés (LACES - EA 4140) - Université Bordeaux 3

\section{Moignard Benjamin et Rubi Stéphanie, « Des dispositifs pour les élèves perturbateurs : les collèges à l'heure de la sous-traitance ? », Carrefours de l'éducation, 2013/2 n 36, p. 47-60}

\section{Introduction}

La question scolaire déborde des cadres de l'école. Viviane Isambert Jamati a bien montré comment la problématique de l'échec scolaire s'est émancipée du seul cadre de l'école pour devenir un «problème social » (Isambert Jamati, 1996). De la même manière aujourd'hui, un ensemble de problèmes interrogeant la gestion des élèves dits «difficiles », interpellent les acteurs éducatifs bien au-delà de la seule sphère de l'école. La mobilisation nouvelle de l'échelon local dans la prise en charge d'un certain nombre de difficultés scolaires favorise la démultiplication des dispositifs et des acteurs intervenants auprès de publics spécifiques. Cet article s'intéresse en particulier aux formes de prise en charge des élèves temporairement exclus, désignés comme élèves perturbateurs, dans des dispositifs externes à l'école. Portés par des professionnels du champ de l'éducation spécialisée, des psychologues, des animateurs socio-culturels, la prise en charge de ces élèves ouvre de nouveaux espaces de relations entre l'école et les partenaires locaux, interrogeant les transformations des modalités et des formes d'intervention autour de ce qui fait difficulté à l'école. Après avoir présenté la nature de ces dispositifs et le contexte de leur mise en place, nous interrogeons les relations liant les partenaires de ces actions, leurs jeux d'influence et de pouvoir au travers de l'explicitation des intentions et des principes de justification afférents (Mangez, 2001). Nous poserons ensuite de manière plus générale la question de savoir si ces dispositifs marquent un tournant dans la prise en charge des élèves désignés comme " perturbateurs", aboutissant à une forme d'externalisation de l'action de l'école, ou si nous assistons plutôt à une mutualisation avérée 
des ressources éducatives disponibles sur un territoire, visant une prise en charge adéquate et une réponse coordonnée d'actions vers les élèves les plus « en difficulté ».

\section{L'émergence des dispositifs assurant la prise en charge d'élèves exclus temporairement : gérer des élèves « perturbateurs » et maintenir l’ordre scolaire}

La gestion de l'ordre scolaire est devenue une préoccupation centrale à mesure que la démocratisation scolaire a entrainé dans son sillage l'accueil de «nouveaux publics » moins préparés aux exigences normatives de l'école. L'école ne fixe plus les règles de manière unilatérale : elle est contrainte par un ordre scolaire qui doit désormais être «négocié » avec les élèves et leurs familles (Périer, 2010). Depuis le milieu des années 1990 et l'attention nouvelle portée à la question des violences scolaires, la préservation d'un espace scolaire protégé s'est imposée comme une priorité nationale, rappelée lors de chacun des multiples plans de lutte contre les violences à l'école. Le temps du chahut anomique intégrateur semble révolu (Testaniere, 1967) au profit de ce qu'Anne Barrère appelle « un nouvel ordre du désordre scolaire », caractérisé en particulier par l'accumulation d'incidents dans l'espace de la classe et de l'établissement, provoqué par la multiplication des conflits relationnels et le développement d'une forme de « désocialisation des problèmes professionnels liés à l'autorité dans l'école » (Barrère, 2002, p. 17). L'école française a vu ses modes de régulation évoluer du fait de cette transformation structurelle liée à la démocratisation scolaire qui pose de manière incontournable la question de la gestion de ces groupes d'élèves « à risque » (Frandji, 2011). C'est dans ce contexte qu'émerge la figure de l'élève dit "perturbateur » que nous définissons comme celui qui remet en cause, par une attitude ou un comportement déviant, l'ordre scolaire établi dans un établissement. L'élève perturbateur est désigné comme tel par les personnels ou une partie des personnels de l'établissement, en fonction de critères normatifs que ces personnels édictent en matière de respect des règles et de reconnaissance de leur autorité.

Divers manquements à l'ordre scolaire sont identifiés par le code de l'éducation qui prévoit des réponses précises déclinées autour d'un certain nombre de sanctions. Parmi elles, l'exclusion des élèves, qu'elle soit temporaire ou définitive, fait figure de réponse la plus grave, marquant l'éviction provisoire ou définitive de l'élève de son établissement. Ces exclusions sont prises en charge de manières diverses par l'institution scolaire, et il serait sans 
doute réducteur d'en donner une vision uniforme. Si maints établissements continuent de gérer cette question de manière interne, force est de constater que de nombreux dispositifs de prise en charge à l'extérieur de l'école se développent conséquemment, notamment autour de la prise en charge des élèves exclus temporairement des collèges.

Cet article s'appuie sur une recherche en cours initiée en janvier $2011^{1}$, autour des dispositifs de prise en charge des élèves temporairement exclus dans 31 villes de trois départements d'Île-de-France ${ }^{2}$. Nous avons identifié de manière exhaustive les 37 dispositifs exclusivement dédiés à la prise en charge des collégiens temporairement exclus sur ce territoire. Ces dispositifs sont affiliés à 85 des 293 établissements que comptent ces 3 départements. Un tiers (29\%) des collèges fait donc appel à l'un de ces dispositifs externalisés d'accueil des collégiens temporairement exclus. Les 208 établissements restants ne recourent pas à ce type de dispositif.

Près de $65 \%$ des établissements étudiés sont inscrits dans des politiques d'éducation prioritaires : Écoles, collèges et lycées pour l'ambition, l'innovation et la réussite (ÉCLAIR) ou Réseau ambition réussite (RAR). Pour cette contribution, nous examinons au travers des propos tenus par les chefs d'établissements, les conseillers principaux d'éducation (CPE) et les responsables des dispositifs, les principes de justification invoqués pour solliciter le dispositif et la place occupée par ce dernier dans le maillage tissé par les acteurs éducatifs du territoire. Nous cherchons ainsi à voir les congruences et les discordances des éléments mis en avant dans la légitimation par les uns et les autres du dispositif. Pour ce faire, nous prenons appui sur 54 entretiens de recherche répartis comme suit : 32 auprès des responsables de dispositifs $(\mathrm{N}=37), 18$ auprès de chefs d'établissement $(\mathrm{N}=85), 4$ auprès de $\mathrm{CPE}^{3}$. À des fins d'historicisation de l'action publique et de contextualisation des propos des acteurs, nous utilisons également l'ensemble des documents de présentation, d'information, de bilan produits pas les dispositifs, les établissements et les collectivités les finançant.

Ces dispositifs se présentent comme une réponse à l'une des préoccupations énoncées comme majeures par les acteurs des établissements dans les nouvelles formes de gestion de

\footnotetext{
1 Une partie de ce travail d'enquête a été menée avec le précieux concours de Myriam Ouafki, doctorante à l'Université Paris-Est-Créteil et chercheuse à l' Observatoire Universitaire International Education et Prévention.

2 Cette recherche bénéficie d'un financement du Fonds social européen et de plusieurs collectivités territoriales.

3 Dans les établissements scolaires, nous avons prioritairement rencontré les acteurs présentés par les collectivités financeurs comme des précurseurs ou des requérants du dispositif.
} 
l'hétérogénéité des élèves, et en particulier de ceux désignés comme «posant des problèmes de comportements » à l'institution scolaire. Nous n'interrogeons pas ici les figures idéaltypiques de l'élève temporairement exclu et de l'élève perturbateur. En revanche, nous confrontons les arguments et justifications émis par les responsables de dispositifs et les chefs d'établissement, et nous étudions les modalités concrètes de prise en charge d'élèves désignés comme perturbateurs. La mise en place de ces dispositifs nous permet de questionner la relation de l'école à ces élèves, les nouvelles formes de prises en charge de ces élèves par une pluralité de professionnels, et les relations et jeux d'influence de ces acteurs qui participent d'une recomposition du paysage institutionnel autour des enjeux scolaires et éducatifs.

\section{Les exclusions temporaires : une ampleur méconnue, mais des motifs semblables}

Le 24 juin 2011 paraît un décret relatif à la discipline dans les établissements d'enseignement du second degré ${ }^{4}$. En sus de la mise en place de l'automaticité des procédures disciplinaires dans certains cas de violences verbales, physiques ou d'autres actes graves, le décret rappelle le « caractère exceptionnel de l'exclusion temporaire qui ne pourra excéder huit jours contre un mois auparavant » (Décret $n^{\circ} 2011-728$ du 24 juin 2011, p. 10876). La loi va donc dans le sens d'un assouplissement du recours à ce type de mesure disciplinaire, et ajoute une nouvelle sanction au travers de « l'exclusion temporaire de la classe » qui ne peut excéder huit jours et nécessite une solution d'accueil interne aux établissements.

Pourtant, très peu des établissements que nous avons étudiés bénéficient en interne d'un système d'accueil spécifique pour les collégiens temporairement exclus, même si les ateliers ou les classes relais ${ }^{5}$ peuvent faire office d'espaces intermédiaires et d'ultime recours disciplinaire avant des exclusions externalisées (Bonnéry, Martin, 2002). Il faut donc le dire clairement : les élèves exclus ne disposent que très rarement d'un encadrement éducatif si des dispositifs hors de l'éducation nationale ne sont pas mis en œuvre. Il est remarquable de constater que malgré les directives du décret de juin 2011, aucun système de comptabilité des exclusions temporaires n'est mis en place par les rectorats de manière à disposer d'informations susceptibles de mieux renseigner l'application des directives ministérielles en la matière. Il est très difficile dans ces conditions de mesurer l'ampleur de ces exclusions et le

\footnotetext{
${ }^{4}$ Décret $n^{\circ} 2011-728$ du 24 juin 2011

${ }^{5}$ Ces dispositifs internes à l'éducation nationale prennent en charge des élèves identifiés comme étant en situation de décrochage scolaire.
} 
nombre d'élèves qu'elles touchent. Il n'existe pas aujourd'hui en France, à la différence d'autres pays européens comme l'Angleterre, l'Allemagne ou la Suède par exemple, de recension précise de ces exclusions temporaires. Les recherches empiriques disponibles sur la question sont elles aussi rares et ne renseignent pas cet aspect malgré d'autres apports de grand intérêt sur les déterminants sexués ou sociaux de la sanction (Ayral, 2011; GrimaultLeprince, Merle, 2008). Une seule étude propose aujourd'hui un chiffrage national de ces exclusions en extrapolant les déclarations de 400 collèges et lycées publics représentatifs : 17000 jeunes seraient définitivement exclus chaque année de leur établissement à l'issue d'un conseil de discipline et plus de 367000 pour un ou plusieurs jours (Fotinos, Horenstein, 2011). Sur la base de ces chiffres, chaque jour de classe, 95 collégiens ou lycéens seraient définitivement exclus de leurs établissements scolaires et plus de 2000 temporairement. Même si cette étude présente des limites et minore sans doute le phénomène, elle a le mérite de tenter de mesurer l'ampleur, massive, d'un phénomène que la puissance publique ne se donne pas les moyens de mieux connaître.

Du coté de notre terrain d'enquête, 1458 élèves exclus temporairement ont été accueillis dans des dispositifs externalisés de leurs collèges en 2011-2012 dans les trois départements concernés. L'ensemble des établissements étudiés reconnaît l'usage régulier de cette sanction qui vise souvent trois groupes distincts d'élèves. À partir des motifs d'exclusion temporaire des élèves dont nous disposons, nous tirons les constats suivants : toutes les exclusions temporaires sont motivées par un problème de comportement, jugé perturbateur et contrevenant à l'ordre scolaire ${ }^{6}$. Les raisons retranscrites et consignées par les dispositifs esquissent trois «profils» d'élèves. Un premier groupe, minoritaire (13\%), est constitué d'élèves qui, de manière exceptionnelle, ont eu un comportement jugé inadapté qui exige selon les personnels une réponse immédiate et significative. Le deuxième groupe représente $18 \%$ de cet échantillon d'élèves exclus temporairement. Leur implication dans un événement jugé grave a mené à une exclusion systématique: lorsque des personnels sont menacés, insultés, et dans de très rares cas agressés, ou lors de bagarres particulièrement violentes. Le troisième groupe d'élèves est majoritaire (69\%). Il est composé d'élèves orientés dans ces dispositifs à la suite de leur implication répétée dans de multiples incidents : l'exclusion marque alors une ultime tentative de réponse après d'autres types de sanctions qui ont souvent été accumulées. Ces élèves accueillis ne correspondent pas forcément aux élèves présupposés

\footnotetext{
${ }^{6}$ A noter cependant que nous considérons seulement ici les élèves qui ont été pris en charge dans les dispositifs externalisés, ce qui ne recouvre pas l'ensemble des exclusions qui concernent un nombre d'élève bien supérieur.
} 
dans la prescription faite par les collectivités. Cet écart entre le public attendu et le public accueilli est un point sensible. Du côté des établissements, plusieurs questions sont aussi à considérer sur la gradation de la perturbation et des sanctions afférentes : où placer le curseur de cette perturbation pour signifier ce qui relève d'un manquement que l'école peut prendre en charge ou ce qui réfère plutôt à une situation qui exige un cadre différent ? On connaît la tendance générale d'individualisation des politiques éducatives et sociales et le développement conséquent des modes de ciblages des élèves qui posent «problèmes » à l'institution scolaire (Astier, 2007). L'enjeu n'est plus tant aujourd'hui de compenser des inégalités sociales ou concentrées sur des territoires particuliers, que de gérer des problèmes sociaux qui se manifestent dans l'école au travers des attitudes, des comportements et des difficultés scolaires que rencontrent en particulier quelques individus (Rochex, 2008). Là encore, la prise en charge des élèves exclus illustre bien cette tendance à une individualisation des réponses qui doit soulager l'institution et qui contourne la question épineuse de l'intégration scolaire d'élèves en tension avec l'univers normatif de l'école. L'exclusion, même temporaire, marque une sanction dont l'ensemble des personnels reconnaît bien souvent le peu de valeur éducative. Elle constitue de fait un risque d'accentuation des difficultés de l'élève que les équipes pédagogiques perçoivent bien. En cela d'ailleurs, nombreux sont les travaux qui rappellent que ces sanctions peuvent renforcer les formes de ruptures de l'élève avec l'école et participer à la construction d'un processus de décrochage (Blaya, 2010 ; Hayden, 2003 ; Millet, Thin, 2005). Les chefs d'établissements comme les personnels « référents 》 auprès des dispositifs, sont quasi unanimes à reconnaître que l'enjeu de ces sanctions n'est pas dans une dimension éducative, mais bien plus dans une acception punitive, visant à normaliser et contraindre les comportements. D'autres concèdent que l'exclusion temporaire est une réponse à l'épuisement professionnel des équipes enseignantes.

Quels qu'en soient les motifs, les collectivités locales ont perçu une forme de défaillance dans la prise en charge éducative par l'institution scolaire de ces élèves, qui les incite à se positionner sur ce nouvel espace d'intervention.

\section{Le positionnement des collectivités locales : territorialisation de l'action éducative et célébration du « local » dans la résolution des difficultés scolaires}

La prise en charge des élèves exclus suscite une attention relativement récente en France alors que d'autres régions du monde ont généralisé des systèmes d'accueil qui visent à prévenir les 
risques de rupture entre l'élève et l'école. Le Québec développe depuis près de 15 ans des dispositifs de «suspension » des élèves dans l'école, en lien avec les travailleurs sociaux du territoire d'implantation des établissements (Massé et al., 2005). L'Australie a développé des programmes de justice restaurative qui visent à prévenir la rupture de l'élève (Wotherspoon et al., 2011). L'Angleterre a concentré certains moyens au travers de la mise en place de programmes comme «Excellence in Cities », visant la réduction de la difficulté scolaire et des exclusions de l'école, ainsi que la promotion de l'inclusion sociale dans les zones les plus défavorisées (Osler et al., 2002). En France, la capacité de mobilisation de l'espace local est présentée depuis les années 1980 comme une ressource indispensable à la modernisation de l'administration publique et à l'amélioration de l'efficacité en éducation, en alimentant d'ailleurs de nombreuses injonctions parfois contradictoires (Pons, 2011). C'est dans ce contexte d'une spatialisation croissante des politiques éducatives qu'émerge ce que Choukri Ben Ayed appelle un «nouvel ordre éducatif local», qui privilégie le développement d'un registre d'intervention appuyé sur les collectivités locales au détriment d'une vision plus égalitariste et républicaine dans l'appréhension de certaines problématiques scolaires (Ben Ayed, 2009).

La prise en charge des élèves temporairement exclus par des structures liées aux collectivités locales relève de ce récent mouvement de relocalisation des formes de gestion de la difficulté scolaire. Si les établissements scolaires continuent de se positionner sur un principe de rupture avec leur environnement immédiat (Moignard, 2008), ils n'hésitent pas à sous-traiter certains aspects de leurs missions auprès des acteurs de ce même environnement. Car il ne faut pas s'y tromper : si des collectivités et des structures socio-éducatives prennent en charge ces élèves, cela ne se fait pas dans un cadre partenarial visant la co-éducation des élèves. Ces dispositifs figurent plutôt la tendance de l'école contemporaine à externaliser la prise en charge de la difficulté scolaire auprès d'acteurs qui y voient des espaces éducatifs voire co-éducatifs à investir.

Sur les 3 départements enquêtés, nous pouvons aujourd'hui dresser un portrait relativement fiable des dispositifs d'accueil des élèves temporairement exclus mis en place. Si quelques dispositifs très spécifiques ont pu nous échapper, le croisement des données à partir des entrées tant par les établissements que par les institutions porteuses des financements nous semble garant d'un certain degré d'exhaustivité. Aux dires de la plupart des «porteurs », il s'agit en général d'accueillir les collégien.ne.s sur leur temps d'exclusion en leur proposant 
des ateliers de remédiation visant une forme de re-socialisation scolaire. Il existe néanmoins quelques dispositifs propres aux établissements souvent insérés dans des dispositifs plus larges tels les ateliers relais. Les dispositifs locaux sont presque systématiquement sollicités directement par les collèges, rarement par des parents ou des travailleurs sociaux. Les exigences des financeurs faisant, les dispositifs sont souvent encadrés par des personnels qualifiés (à bac plus deux au moins), et issus, pour la plupart, de l'éducation spécialisée, avec une forte présence de psychologues qui dirigent près de la moitié des 37 dispositifs répertoriés. Le coordinateur du dispositif est fréquemment secondé par une ou deux autres personnes, elles-mêmes entourées d'intervenants extérieurs en charge de la mise en place d'ateliers spécifiques autour d'activités sportives, culturelles ou artistiques servant des intentions présentées autour de la citoyenneté. Plus de $82 \%$ des dispositifs que nous avons recensés sont «portés » par des services municipaux : service Politique de la Ville pour un peu plus de $12 \%$, service d'action sociale, de prévention pour $16 \%$, alors que plus de $54 \%$ dépendent de programmes de réussite éducative (PRE). Un peu moins de $18 \%$ des dispositifs sont conduits par des associations de jeunesse ou d'insertion qui bénéficient alors pour cette action de financements municipaux ou départementaux dédiés à l'accueil des élèves exclus. On trouve donc une certaine diversité dans les types de structures partenaires, avec une forte présence de la réussite éducative (PRE) sur un certain nombre de territoires, et qui peut dès lors user de cette opportune implantation.

Du côté des établissements, l'exclusion temporaire fait très fréquemment figure de moyen de gestion interne, voire de levier possible dans les modes de gouvernance. Elle révèle une exigence de crédibilité de la part des équipes de direction et des équipes de vie scolaire, dans leur capacité à apporter une réponse concrète à un problème sinon insoluble. La réponse « éducative » leur apparaît non seulement insuffisante, mais aussi compromise par son manque d'efficacité supposée, et l'on retrouve là une forme de remise en ordre de l'institution que Bertrand Geay a bien illustrée à partir de l'exemple des conseils de discipline (Geay et al., 2009). L'exclusion temporaire est donc d'abord un outil de régulation interne dans un contexte où la multiplication des incidents scolaires affaiblit les enseignants dans leur légitimité professionnelle, et nécessite des recours symboliques de restauration de leur autorité. Au-delà d'un manque de moyens dédiés à la prise en charge de certains de ces élèves, cet enjeu lié aux professionnalités est l'une des pistes pour comprendre le peu d'établissements impliqués dans la mise en place de dispositifs d'accueil internalisés à l'occasion des exclusions temporaires. C'est bien dans ce contexte que d'autres institutions se 
positionnent pour prendre en charge ces élèves. Les principes de justifications inférés à ces dispositifs par les acteurs concernés permettent de faire émerger les articulations et les arrangements entre divers ordres locaux: aux nécessités de gouvernance d'équipes enseignantes lasses et très réactives, d'établissements dés-ordonnancés par des comportements parfois extrêmes d'élèves, correspondent des enjeux et des logiques d'autres acteurs locaux investis dans les questions éducatives et sensibles à la mobilisation de référentiels psychologiques dans la prise en charge des élèves.

\section{La conversion de problématiques scolaires en problématiques psychiques : un espace consensuel}

La mobilisation des collectivités et des porteurs de dispositifs dans l'accueil des élèves exclus temporairement répond à une vocation éducative largement affirmée. Reste qu'avec ces prises en charge, les professionnels qui animent ces dispositifs touchent un public auquel ils auraient sinon difficilement accès.

Mme Brêma, Coordinatrice dispositif, Directrice politique de la ville : ça peut faire un relais. Alors nous, on a le dispositif d'accueil, ça nous a permis en fait, je crois que l'année dernière on a eu trois suivis, on a détecté, parce que le collège ne se saisit pas du dispositif PRE... ça nous a, en tout cas, permis de détecter des élèves qui venaient pendant une semaine de se dire voilà, ces élèves là sont éligibles au PRE et on va continuer à travailler avec eux. Parce qu'autant les écoles élémentaires, les écoles maternelles, on est 88 enfants sur le PRE, ils font énormément appel au dispositif, autant les collèges, on a très très peu... Donc sur ce dispositif là c'est vrai qu'il y a très peu de collèges qui s'en saisissaient, et du coup ça nous permettait nous, à travers les situations qu'on rencontre dans la prise en charge des élèves exclus, de pouvoir continuer un travail avec les élèves qui le souhaitent. En tout cas on leur présente le PRE et ils s'inscrivent ou ils s'inscrivent pas. Là je sais qu'on a sur les élèves de l'année dernière on a eu trois suivis, enfin trois entrées sur le PRE.

Il n'est ainsi pas rare que les collégiens exclus participent suite à leur passage au dispositif d'accueil à d'autres actions des «porteurs », dont un peu plus d'un tiers assurent qu'ils ne pourraient pas mener d'autres actions auprès des mêmes collégiens sans le dispositif d'accueil des collégiens exclus temporairement. Leur principe de justification prône une attention au jeune différente, non stigmatisante dans le cadre du dispositif qui est aussi lu comme un « tremplin » pour les intégrer dans d'autres dispositifs. Le "portage» de ces dispositifs 
d'accueil des élèves temporairement exclus par des acteurs extérieurs à l'école et leur financement par les collectivités locales sont justifiés par deux types d'arguments. D'abord, la nécessité d'une prise en charge d'élèves susceptibles de perturber l'ordre public et d'entrer dans des processus de décrochage coûteux pour les collectivités, en particulier sur le registre de l'entrée dans des carrières délinquantes (Esterle-Hedibel, 2006). Ensuite, on observe une volonté de diversification de l'activité des intervenants sociaux qui est sans doute à mettre en lien avec le morcellement et l'éparpillement de l'intervention sociale dont l'action n'est plus circonscrite aux seules missions traditionnelles du travail social (Ion, 2005). Nous faisons même l'hypothèse que la prise en charge de difficultés scolaires est devenue un enjeu majeur pour nombre de structures du champ de l'intervention sociale, et en particulier de celles qui interviennent sur un principe de proximité avec un territoire. La multiplication des espaces dédiés à l'accompagnement scolaire ou le développement des projets partenariaux visant à prévenir le décrochage scolaire par exemple, mobilisent très largement les acteurs locaux. Les collectivités s'engagent même parfois au-delà de leurs obligations légales sur ces questions : c'est le cas par exemple de la prise en charge financière significative des dispositifs d'accueil des collégiens exclus temporairement par des conseils généraux sur de nombreux dispositifs étudiés. L'Éducation nationale de son côté ne dégage aucun moyen pour ces dispositifs, malgré l'obligation qui est faite aux établissements selon 1'article L.131-6 du Code de l'éducation, de proposer aux élèves une mesure destinée à leur garantir un suivi éducatif, la poursuite de la scolarité et la facilitation de la réintégration après leur exclusion. Plus encore, les enseignants ne sont qu'exceptionnellement en contact avec les encadrants de ces dispositifs, dont ils ne connaissent même pas l'existence en général. Dès lors, l'enjeu pourtant affiché de la continuité scolaire est assez peu pris en compte dans ces dispositifs qui par nature, vont plutôt privilégier d'autres registres d'intervention que ceux de la remobilisation scolaire traditionnelle, très éloignée des cultures professionnelles des intervenants mobilisés.

Les dispositifs se font fort de palier ce manque d'encadrement éducatif en axant une part importante de l'intervention sur des enjeux de resocialisation scolaire et un début de prise en charge psychologique. Ce dernier aspect est prégnant, voire écrasant, dans de nombreux dispositifs et fait même partie des attentes des financeurs dans la plupart des cas. Il est d'ailleurs frappant de constater la manière avec laquelle les problèmes psychologiques voire psychiatriques sont avancés dans les propos tenus par les "porteurs », mais aussi dans ceux des principaux qui se positionnent alors comme prescripteurs légitimes : 
Mme Grison, chef de projet PRE, coordinatrice dispositif : " nous en 2008 on a dit "on va commencer par une petite porte", bon ba maintenant la porte elle commence vraiment à être trop petite puisque là encore ce matin, je suis en train de faire des bilans pour le second trimestre, la plupart ont besoin de voir au moins un psy, au moins un SAS (Service d'Accueil Spécialisé), le CMPP (Centre Médico-Psycho-Pédagogique) ça fait peur, donc nous c'est le Clobénart qui est la psychiatrie adolescent pour le territoire, j'vous en parle même pas! alors qu'on a des problèmes de santé mentale, de santé psychologique, souffrance psychologique importante, déstructurations familiales lourdes... les collèges en sont conscients, et même les collèges disent "Bon ok la continuité scolaire d'accord mais, y'a un moment donné, si l'acte a été posé, faut..." Donc en fait, j'ai envie de dire, que c'est le haut de l'iceberg quand ils arrivent ici... (...) Quand on arrive au comité de pilotage et qu'on a les principaux qui en gros nous demandent de faire une $2^{e}$ classe-relais, pourquoi pas de les soutenir sur ça mais dans la discussion ils sont sur "il faut aller plus sur la santé mentale, il faut aller plus sur la santé psy, ils sont trop fragiles". ».

M. Tä̈aut, principal : " On la faisait très discrètement la prise en charge. $A$ l'heure actuelle, elle se fait sur des élèves qui présentent des difficultés d'ordre "troubles du caractère" ou "du comportement" quoi. Plutôt cette dimension là que grand perturbateur. (...) En fait, pour caricaturer, le profil d'élèves difficiles du type "milieu social défavorisé", "structure familiale déstructurée", si le gamin arrive lui-même plus ou moins délinquant et qu'il s'oppose plus ou moins à l'institution quand il est au collège, c'est pas notre cœur de cible pour ce dispositif. D'ailleurs, les tentatives n'ont pas forcément marché avec ces élèves-là. On a plutôt des élèves avec des parents plutôt attentifs, et donc un élève avec un trouble du comportement ou de la conduite. ».

De fait, les attentes en terme de continuité scolaire sont relativement restreintes de la part des porteurs de dispositifs comme des chefs d'établissements. L'Éducation nationale affiche pourtant une intransigeance très forte sur cette question, allant parfois jusqu'à recommander le référencement des apprentissages travaillés dans le cadre du dispositif au regard du socle commun de compétence. Mais force est de constater qu'il s'agit d'abord d'une posture formelle, puisque l'immense majorité des dispositifs ne disposent pas d'éléments de la part des établissements leur permettant d'assurer un minimum de continuité scolaire. Nombreux sont d'ailleurs les porteurs de dispositifs à pointer cette limite : si les accueils permettent de recevoir les élèves dans un espace dédié et encadré par des professionnels qui leur semble préférable à une mise à la rue, les difficultés que ces élèves rencontrent souvent du point de vue des apprentissages, ne sauraient être résolus par moins d'école. En revanche, émerge une attention très forte de la part des prescripteurs que sont les financeurs et les chefs d'établissements, pour une forme de prise en charge psychologique des élèves, à partir du placement dans ces dispositifs. Près de $75 \%$ des dispositifs étudiés font référence dans leurs 
activités à des temps substantiels dédiés à une prise en charge individuelle des élèves qui dépasse largement le cadre de la sanction liée à la présence de l'élève dans le dispositif. Huit dispositifs énoncent ainsi dans leur protocole de prise en charge une démarche permettant d'identifier d'éventuelles pathologies, et de nombreux autres y travaillent de manière moins formelle. Paradoxalement ou non d'ailleurs, ce sont quelques dispositifs coordonnés par des psychologues qui affichent le plus de réserves sur ces formes de prise en charge qu'ils dénoncent comme "psychologisantes », alors qu'ils insistent sur la nécessité de se recentrer sur les questions scolaires et le besoin de renforcement de l'estime de soi des élèves à la suite de ces sanctions. Ainsi, dans les expérimentations premières et fondatrices du modèle, généralisées par la suite à l'échelle de départements entiers, ces espaces de prise en charge des élèves exclus temporairement visaient prioritairement un travail autour de la sanction. $\mathrm{Ni}$ dispositif conçu pour les élèves « décrocheurs », ni espace pensé pour les élèves en situation d'échec scolaire, dans les récits fondateurs dont sont détenteurs certains des premiers « porteurs » et quelques personnes en charge de ces dispositifs au sein des collectivités et des directions académiques, l'accueil de ces élèves s'ancrait bien plus dans une volonté d'agir sur le métier d'élève, sur l'hexis scolaire attendue et prescrite par l'institution, sur les relations de l'élève aux autres, que sur une nécessité de prise en charge psychologique. Cette tendance à la mobilisation du registre psychologique pour traiter de problématiques liées d'abord à une confrontation à l'ordre scolaire et à la lecture qui en est faite par des équipes souvent sous tension, renvoie aussi à une forme de pathologisation des problématiques sociales qu'il faut pouvoir interroger.

\section{Conclusion}

Le « dispositif » devient un outil essentiel de la gestion d'un certain nombre de difficultés scolaires. Si la mobilisation de l'échelon local marque une implication renforcée des collectivités et des structures socioéducatives dans la prise en charge des élèves les plus en difficultés, c'est bien plus la tendance à une forme d'externalisation par l'école de certaines de ses problèmes qui nous semble marquer une évolution significative des rapports de l'institution scolaire à son environnement. Nous sommes revenus sur la genèse de dispositifs qui s'inscrivent dans un contexte de confrontation des univers normatifs juvéniles et scolaires, 
où la remise en cause de l'ordre scolaire suscite des réactions virulentes des équipes que les exclusions temporaires illustrent bien. Du coté des collectivités et des associations, la présence de ces adolescents exclus temporairement dans l'espace public appelle à un impératif de gestion liée tout autant à une vocation éducative affirmée, un enjeu de prévention des risques de glissement de ces élèves vers des activités délinquantes, et l'occasion de toucher des jeunes sinon difficilement atteignables par les structures traditionnelles. Mais là où nous pourrions lire une occasion de co-éducation qui viendrait renforcer l'école dans sa capacité de prise en charge de tous les élèves, nous observons plutôt une externalisation par l'école de la gestion de ces élèves qui ne correspondent pas à son modèle pédagogique. Cette externalisation pose le risque du développement des espaces supplétifs tentant de pallier les difficultés de l'institution scolaire, là où les exigences d'une école démocratique appellent à un traitement égalitaire des élèves. Reconnaissants les besoins d'accompagnement et de suivis spécifiques et adaptés pour certains élèves, l'ampleur de ces dispositifs, qui concernent un nombre important d'élèves et d'établissements dans plusieurs départements, nous amène à interroger le risque d'une pathologisation des problèmes sociaux que l'école ne parviendrait pas, comme d'autres institutions, à dépasser (Coutant, 2012). La tendance à la personnalisation de l'accompagnement des élèves pour traiter l'hétérogénéité des publics et la mobilisation systématique du registre psychologique pour apprécier des problèmes d'abord scolaires ou plus largement sociétaux, marquent une démultiplication des espaces susceptibles de répondre à chacune des problématiques décelées. Ce mouvement alimente des formes d'interdépendance entre les dispositifs en termes de gestion de flux, d'agencement des uns par rapport aux autres, et par là même, renforce et entérine le morcellement de l'action éducative. Dès lors, le jeune accueilli devient un sujet fragmenté, à qui est interdit le statut d'élève, au profit de l'entrée dans une carrière de bénéficiaires et d'usagers de dispositifs multiples, qui l'éloignent un peu plus encore des exigences de l'école. Une telle situation questionne les processus de désubjectivation internes aux dispositifs (Agamben, 2006), qui maintiennent les individus dans des rôles sociaux assignés d'élèves perturbateurs que l'école ne pourrait plus prendre en charge. Pourtant, ces dispositifs peuvent constituer une ressource pour que l'école assure son ambitieuse mission républicaine. Mais sans doute faudrait-il alors préférer à une logique d'externalisation des difficultés, une capacité de mobilisation sur un registre partenarial qui intègre les exigences indispensables de la continuité scolaire et de l'accueil des élèves dans une école bien-traitante. Les dispositifs de ce type peuvent sans doute constituer, dans certaines conditions, une partie de la solution aux problèmes difficiles qui traversent aujourd'hui l'école. Si certains adolescents doivent apprendre à redevenir élèves, l'école ne 
doit pas perde de vue que c'est à elle que revient la mission essentielle d'assurer l'instruction et l'éducation des citoyens de plein droit qu'ils sont déjà.

Agamben G. (2006). Qu'est ce qu'un dispositif? Paris : Payot et Rivages.

Astier I. (2007). Les nouvelles règles du social. Paris : PUF.

Ayral S. (2011). La fabrique des garçons. Sanctions et genre au collège, Paris : PUF.

Barrère A. (2002). Un nouvel âge du désordre scolaire : les enseignants face aux incidents. Déviance et Société, n 26, p. 3-19.

Ben Ayed C. (2009). Le nouvel ordre éducatif local. Mixité, disparités, lutte locale. Paris : PUF.

Bonnéry S., Martin E. (2002). Classes relais. Un dispositif pour les élèves en rupture avec l'école. Paris : ESF.

Blaya C. (2010). Décrochage scolaire. L'école en difficulté. Bruxelles : De Boeck.

Coutant I. (2012). Troubles en psychiatrie : Enquête dans une unité pour adolescents, Paris : La Dispute.

Esterle-Hedibel M. (2006). Absentéisme, déscolarisation, décrochage scolaire, les apports des recherches récentes. Déviance et Société, n 30/1, p. 41-65.

Fotinos G., Horenstein J.-M. (2011). La qualité de vie au travail dans les lycées et les collèges. Rapport MGEN.

Frandji D. (2011). "Groupes à risque", "besoins spécifiques" : raison gestionnaire et ghetto épistémologique au service d'une redéfinition scolaire ? in M. Demeuse, D. Frandji, D. Greger, J.-Y. Rochex Les politiques d'éducation prioritaire en Europe. Tome 2. Quel devenir pour l'égalité scolaire? Paris : ENS éditions, p. 169-209.

Geay B., Oria N., Fromard L. (2009). La remise en ordre symbolique de l'institution. Les conseils de discipline dans l'enseignement secondaire. Actes de la recherche en sciences sociales, $\mathrm{n}^{\circ} 178$, p. 62-79.

Grimault-Leprince A., Merle P. (2008). Les sanctions au collège. Les déterminants sociaux de la sanction et leur interprétation. Revue française de Sociologie, ${ }^{\circ} 49$, p. 231-267.

Hayden C. (2003). Responding to Exclusion from School. Journal of Educational Administration, $\mathrm{n}^{\circ}$ 41, p. 626-639.

Ion J. (2005). Le travail social à l'épreuve du territoire. Paris : Dunod.

Isambert-Jamati, V. (1996). Quelques rappels de l'émergence de l'échec scolaire comme " problème social" dans les milieux pédagogiques français, Migrants-Formation, $\mathrm{n}^{\circ} 104$, mars, p. 28-40.

Mangez É. (2001). Régulation de l'action éducative dans les années quatre-vingt dix, Éducation et sociétés, $2, \mathrm{n}^{\circ} 8$, p. 81-96. 
Massé L., Desbiens N., Lanaris C. (2005). Les troubles du comportement à l'école : Prévention, évaluation et intervention. Montréal : Gaëtan Morin.

Millet M., Thin D. (2005). Ruptures scolaires : l'école à l'épreuve de la question sociale. Paris : PUF.

Moignard B. (2008). L'école et la rue : fabriques de délinquance. Paris : PUF.

Osler A., Street C., Lall M., Vincent K. (2002). Not a Problem ? Girls and School Exclusion. London : National Children's Bureau.

Périer P. (2010). L'ordre scolaire négocié. Parents, élèves, professeurs dans les contextes difficiles. Rennes : PUR.

Pons X. (2011). L'évaluation des politiques éducatives. Paris : PUF.

Rochex J.-Y. (2008). Les politiques d'éducation prioritaire en Europe, d'un "âge" et d'un pays à l'autre. In M. Demeuse, D. Frandji, D. Greger, J.-Y. Rochex. Les politiques d'éducation prioritaire en Europe. Tome 2. Quel devenir pour l'égalité scolaire? Paris : ENS éditions. p. 409-451.

Testaniere J. (1967). Chahut traditionnel et chahut anomique dans l'enseignement secondaire. Revue francaise de sociologie, $\mathrm{n}^{\circ}$ 8, p. 17-33.

Wotherspoon A.J., Cox G., Slee P.T. (2011). Working outside of the boundaries in R. Shute Mental health et wellbeing: Educational perspectives. Adelaide SA: Shannon Research Press, p. 275-284. 